Bangladesh J. Plant Taxon. 15(2): 115-128, 2008 (December)

(C) 2008 Bangladesh Association of Plant Taxonomists

\title{
SYSTEMATIC STUDIES IN THE FAMILY LILIACEAE FROM BANGLADESH
}

\author{
Sumona Afroz ${ }^{1}$ and Md. Abul Hassan \\ Department of Botany, University of Dhaka, Dhaka 1000, Bangladesh
}

Keywords: Liliaceae, Systematic studies, Bangladesh

\begin{abstract}
The family Liliaceae A.L. de Jussieu has been revised for Bangladesh and a total of 34 species with one variant under 16 genera have been recorded. Artificial dichotomous keys to the genera and species have been given. Descriptions have been provided for each taxon, and local names, flowering and fruiting periods have been added wherever available. Out of 34 species, 16 species are native/naturalized and 18 species, including 1 variant, are exotic. Four genera, ten species and one variant have been documented for the first time in Bangladesh.
\end{abstract}

\section{Introduction}

Liliaceae A.L. de Jussieu, the Lily family, is a moderately large family consisting of about 280 genera and nearly 4000 species, widespread throughout the world, but most abundant and varied in fairly dry, temperate to subtropical regions. The family is characterized by the following diagnostic characters: i) Perennial or annual herbs, rarely shrubs, with starchy rhizome, bulb or corm; ii) Leaves simple, alternate or less often opposite or whorled, often all basal; iii) Flowers in a raceme, spike, panicle or involucrate cymose umbel, sometimes solitary or paired in the axils of the leaves; iv) Tepals 6-8, usually in 2 similar petaloid cycles, stamens usually as many as the tepals; v) Carpels 3 (rarely 2 or 4), united, ovary superior or inferior with axile or basal placentation; vi) Fruit a loculicidal or septicidal capsule, less often a berry, seeds often flat (Cronquist 1981).

Many taxonomists, viz. Bentham and Hooker (1862-1883), Engler and Prantl (18871915), Bessey (1915), Rendle (1925), and Hutchinson (1934) described Amaryllidaceae (ovary inferior) and Liliaceae (ovary superior) separately under the same order or under separate orders mainly on the basis of ovary position, but other characters are more or less the same. So it is more logical to keep both the families under a single larger family as done by Cronquist (1981) and Takhtajan (1980). The same treatment is followed here.

Species of this family are much important for economic uses. Most of the species are poisonous for cattle, but there are many species in this family which are commercially useful for their medicinal value.

${ }^{1}$ Corresponding author. E-mail: binidu@yahoo.com 
A general account of Liliaceae (including Amaryllidaceae) occurring in Indian subcontinent was given by Hooker (1892) and for the then Bengal by Prain (1903). In the present context, these accounts are not up to date regarding the number of taxa as well as nomenclature. After Hooker and Prain's publications many floras on the smaller areas of present Bangladesh have been published where the authors included Liliaceae and Amaryllidaceae either separately or jointly under Liliaceae, viz. Heinig (1925), Cowan (1926), Raizada (1941), Datta and Mitra (1953), Sinclair (1955), Mia and Khan (1995), Uddin et al. (1998), Rahman (2004), and Uddin and Hassan (2004). However, there has been no revisionary work on Liliaceae for Bangladesh.

\section{Materials and Methods}

The present work is mainly based on the fresh materials collected by the first author. The work is also supplemented by the herbarium specimens housed at the Dhaka University Herbarium (DUH) and Bangladesh National Herbarium (DACB). The germplasm of most of the taxa have been collected and planted in the experimental plots of the Dhaka University Botanical Garden for further studies.

The collected specimens were identified consulting standard literature like Hooker (1892), Jackson (1895), Prain (1903), Dassanayake and Clayton (1981), and Deb (1983), and comparing with herbarium specimens available both in the DUH and DACB.

The present work is intended to record all the species growing all over Bangladesh in the wild and also cultivated in the garden and in the field and to make a revision of the family for the country. Short descriptions, important synonyms, precise localities and local names of each species are given wherever available along with the correct botanical names. Flowering and fruiting periods $(F l$. \& Fr.) are also noted. The genera and species are arranged alphabetically.

\section{Results and Discussion}

The systematic studies in the family Liliaceae from Bangladesh resulted in the confirmation of 34 species and a variant under 16 genera, whereas Hooker (1892) reported only 17 species under 10 genera and Prain (1903) mentioned 14 species under 11 genera in greater Liliaceae for the present Bangladesh area. For easy identification, dichotomous keys to the genera and also to the species have been constructed on the basis of easily recognizable characters.

Four genera (Haemanthus, Hippeastrum, Proiphys and Eucharis), eleven species (Asparagus densiflorus, A. officinalis, A. setaceus, Crinum jagus, Eucharis grandiflora, Haemanthus multiflorus, Hippeastrum $\times$ johnsonii, Proiphys amboinensis, Zephyranthes atamasco, Z. candida, and Z. grandiflora), and one variant of Crinum asiaticum, most of which are planted in gardens, have been documented for the first time in Bangladesh. 


\section{Key to genera}

1. Inflorescence surrounded by involucral bracts or spathes 2

- Inflorescence not surrounded by involucral bracts $\quad \mathbf{9}$

2. Scape 1-flowered

- Scape more than 1-flowered

3. Leaves radical, scapose

- Leaves not scapose, usually strap shaped

Hippeastrum

5. Flowers without staminal corona 6

- Flowers with staminal corona $\quad 7$

6. Leaves usually evergreen

- Leaves appearing along with or after the appearance of scape

7. Leaves without petiole; corona soft

- Leaves with petiole; corona hard and waxy

8. Corona 6-partite; scape more than 10-flowered

- Corona united; scape up to 10-flowered

9. Leaves reduced to minute scales, often spinescent, bearing in their axils tufts of leaf-like cladodes

- Leaves simple

10. Inflorescence branched

- Inflorescence unbranched

11. Fruit indehiscent

- Fruit dehiscent

12. Perianth tube produced above the ovary; stamens perigynous

- Perianth tube not produced above the ovary; stamens epigynous

13. Aerial annual stem climbing, leafy; leaves with tendril-like tips

- Aerial annual stem or scape erect, leafless

14. Inflorescence 1 or 2-flowered

- Inflorescence many-flowered

15. Perianth campanulate; seeds many

- Perianth not campanulate; seed usually 1
Zephyranthes

3

Allium

4

5

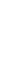

\section{Crinum}

Haemanthus

Pancratium

8

Proiphys

Eucharis

Asparagus

10

Hemerocallis

11

12

13

Curculigo

Molineria

Gloriosa

14

Hypoxis

15

Urginea

Asphodelus 


\section{Systematic enumeration of taxa}

Allium [Tourn.] Linn., Syst. ed. 1 (1735).

\section{Key to species}

1. Leaves fistular

A. cepa

- Leaves flat

2

2. Bulb long, cylindric, simple; heads bearing flowers only

A. tuberosum

- $\quad$ Bulb short, compound; heads bearing both flowers and bulbils

A. sativum

1. Allium cepa L., Sp. Pl. ed. 1: 300 (1753).

Local name: Peyaj.

An annual herb. Leaves fistular, heads dense. Flowers white with mid-green line in tepals. Fruit a capsule. Seeds compressed, black. Fl. \& Fr.: February-June. Cultivated.

Specimens examined: Dhaka: Dhaka University Botanical Garden, 23.4.1940, Atul (DUH); 11.1.2007, Sumona 6 (DUH); 19.3.2007, Sumona 9 (DUH).

2. Allium sativum L., Sp. P1. 1: 296 (1753).

Local name: Rashun.

An erect, annual herb. Leaves flat, heads bearing both flowers and bulbils. Flowers greenish-white. Fruits seedless. Fl. \& Fr.: February-April. Cultivated.

Specimen examined: Munsigonj: Shirajdikhan, 23.3.2008, Sumona 48 (DUH).

3. Allium tuberosum Rottler ex Spreng., Syst. 2: 38 (1825). A. uliginosum G. Don, Mem. Wern. Soc. 6: 60 (1827).

Local name: Banga gandina.

An erect, annual herb. Leaves flat, heads bearing only flowers. Flowers white or pink. Fruits obcordate. Fl. \& Fr.: June-August. Cultivated.

Specimen examined: Maulvi Bazar: Srimangal (Khasia Palli), 25.8.1998, M. Yusuf 1062 (BCSIR Lab.).

\section{Key to species}

1. Branchlets and cladodes arranged like a fern frond 2

- Branchlets and cladodes not arranged like a fern frond

2. Inflorescence 5-9 flowered; cladodes 2-9 per node

- Inflorescence 1-4 flowered; cladodes 10-15 per node

3. Stem not terete, rather triquetrous

- Stem terete

4. Flowers pendulous, solitary or in pairs in the leaf axils 3

- Flowers not pendulous, more than 2 flowers cluster in the leaf axils

\section{A. densiflorus \\ A. setaceus \\ A. acerosus \\ 4}

A. officinalis

5 
5. Spines straight

\section{A. adscendens}

- $\quad$ Spines reflexed

\section{A. racemosus}

4. Asparagus acerosus Roxb., Fl. Ind. 2: 150 (1832). Asparagopsis acerosa Kunth, Enum. P1. 5: 102 (1850).

Local name: Shatamuli.

A perennial, sub-scandent undershrub. Stem triquetrous. Flowers white. Fruit a berry, 1-seeded. Fl. \& Fr.: November-March. Native/naturalized.

Specimens examined: Tangail: Madhupur, 29.6.1969, Alo Rani 306 (DUH); Madhupur, 29.6.1969, Asiya 118 (DUH); Madhupur, 29.6.1969, Panna 115 (DUH); Madhupur, 22.12.1982, Mia 892 (DACB); Madhupur, 28.2.1987, Huq et al. 8215 (DACB).

5. Asparagus adscendens Roxb., F1. Ind. 2: 153 (1832). Asparagopsis adscendens Kunth, Enum. Pl. 5: 102 (1850).

Local name: Shatamuli.

A dioecious, evergreen shrub with white tuberous roots. Stem terete, spines straight. Fruit a berry, 1-seeded. Native/naturalized.

Specimen examined: Gazipur: Sal forest, 10.12.1944, Badal Singh (DUH).

6. Asparagus densiflorus (Kunth) J.P. Jessop, Bothalia 9: 51 (1966). Asparagopsis densiflora Kunth, Enum. Pl. 5: 96 (1850); Asparagus sprengeri Regel, Act. Hort. Petrop. 11: 302 (1890); Protasparagus densiflorus (Kunth) A.A. Oberm., Fl. South Afr. 5(3): 49 (1992).

An evergreen, perennial herb with more or less tuberous roots. Flowers greenishwhite with orange anthers. Fruit a bright-red berry, 3-seeded. Fl. \& Fr.: February-April. Cultivated.

Specimens examined: Dhaka: Baldha Garden, 23.3.2007, Sumona 11 (DUH); Baldha Garden 26.4.2007, Sumona 23 (DUH).

7. Asparagus officinalis L., Sp. Pl.: 313 (1753).

A dioecious, erect, perennial herb with a robust tuberous rhizome. Flowers white or pink. Fruit a globose berry, red, 1-6-seeded. Fl. \& Fr.: March-July. Cultivated.

Specimen examined: Dhaka: Dhaka University Botanical Garden, 15.5.2008, Sumona 49 (DUH).

8. Asparagus racemosus Willd., Sp. Pl. 2: 152 (1799).

Local names: Satmuli, Shatamuli, Hilungbupang (Tippara). 
A perennial, slender, scandent or twinning shrub. Stem terete, spines reflexed. Flowers white with purplish anthers and sweet scented. Fruit a globose berry, turn red when ripe, 1-seeded. Fl. \& Fr.: November-March. Native/naturalized.

Specimens examined: Dhaka: Dhaka University Botanical Garden, 5.2.1983, M.A. Hassan 501 (DUH); Dhaka University Campus (Science Library), 4.2.2006, Sumona 1 (DUH); Dhaka University Campus (Ekushey Hall), 2.12.2006, Sumona 4 (DUH); Dhaka University Botanical Garden, 21.5.2007, Sumona 34 (DUH).

9. Asparagus setaceus (Kunth) J.P. Jessop, Bothalia 9: 51 (1966). Asparagopsis setacea Kunth, Enum. Pl. 5: 82 (1850); Asparagus plumosus Baker, J. Linn. Soc. 14: 613 (1875).

Local name: Fern asparagus.

A woody vine, branchlets and cladodes arranged like a fern frond. Flowers white, fragrant. Fruit a berry, purplish-black, 1-3-seeded. Fl. \& Fr.: February-June. Cultivated.

Specimens examined: Dhaka: Nazimuddin Road, 20.1.1956, Shajahan (DUH); Baldha Garden, 17.2.1988, Rezia et al. 249 (DACB).

Asphodelus [Tourn.] Linn., Syst. ed. 1 (1735).

10. Asphodelus tenuifolius Cavan, Anal. Cienc. Nat. 3: 46, t. 27 (1801). A. fistulosus L., Sp. Pl.: 309 (1753); A. parviflorus Wight, Ic. t. 2062 (1853).

An annual herb. Flowers white with a brownish costa. Fruit a globose capsule. Seeds trigonal with 3-4 dorsal ridges. Fl. \& Fr.: May-July. Native/naturalized.

Specimens examined: Chuadanga: Chuadanga, 2.1.1976, Huq, Rahman and Mia 1758 (DACB). Kushtia: 24.4.1957, M.Q. Kabir 52 (DUH). Narayanganj: Postogola, 21.5.1970, M.A. Rahman 133 (DUH). Rajshahi: Biraldah, 5.2.1973, Huq 778 (DACB).

Crinum L., Gen. Pl. ed. 1: 97 (1737); Sp. Pl.: 291 (1753).

\section{Key to species}

1. Perianth tube straight, erect

- Perianth tube curved, declinate

2. Umbels more than 15-flowered

- Umbels up to 15-flowered

3. Leaf margin scabrous; perianth vertically reddish on the back

- Leaf margin smooth; perianth white

4. Scape and perianth tube green

- $\quad$ Scape and perianth tube purplish

5. Bulbs with a fusiform, stoloniferous base; perianth lobes linear

- Bulbs not stoloniferous; perianth lobes lanceolate
2

3

4

5

C. latifolium

C. jagus

C. asiaticum

C. asiaticum, a variant

C. defixum

6 
6. Leaves acuminate, scabrous; perianth lobes shorter than the tube

C. amoenum

- Leaves obtuse or sub-acute; perianth lobes longer than the tube

C. pratense

11. Crinum amoenum Roxb., Fl. Ind. 2: 127 (1832).

Local name: Gang kachu.

A bulbous perennial herb. Leaf margin scabrous. Flowers white, filament purple in upper half portion. Fruit a capsule. Fl. \& Fr.: May-August. Native/naturalized.

Specimens examined: Dhaka: Uttara (Sector-5), 24.5.2007, Sumona 36 (DUH); Baldha Garden, 26.5.2007, Sumona 38 (DUH).

12. Crinum asiaticum L., Sp. Pl.: 292 (1753). Crinum toxicarium Roxb., Fl. Ind. 2: 134 (1832).

Local names: Bara kanur, Gaerhonar pata, Kachori, Nagdal, Sukhdarshan.

A perennial herb. Flowers white. Fruit a capsule, green. Seeds round, concave. Fl. \& Fr.: March-November. Treated here as Crinum asiaticum L., proper. Chromosome no.: $2 \mathrm{n}=22$ (Lubna et al. 2004). Native/naturalized.

Specimens examined: Dhaka: Dhaka University Botanical Garden, 1.7.1968, Mozahar 155 (DUH); Uttara (Sector-3), 12.7.2007, Sumona 43 (DUH); Dhaka University Botanical Garden, 8.8.2007, Sumona 41 (DUH). Jhalakathi: Chankati, 3.3.1987, Huq and Mia 6667 (DACB).

13. Crinum asiaticum L., a variant.

Local name: Sukhdarshan.

A perennial herb. Flowers purplish. Fruit and seed were not found. Fl. \& Fr.: almost throughout the year. Chromosome no.: $2 \mathrm{n}=33$ (Lubna et al. 2004). Cultivated. It is perhaps a triploid plant.

Specimen examined: Dhaka: Dhaka University Botanical Garden, 15.11.2006, Sumona 3 (DUH).

14. Crinum defixum Ker-Gawl., Quart. Journ. Sci. 3: 105 (1817). C. asiaticum Roxb., Hort. Beng. 23 (1814).

Local name: Sukhdarshan.

A very stout, bulbous herb. Flowers white, perianth tube straight, erect. Fruit an ellipsoid capsule, 1-2-seeded. Fl. \& Fr.: May-August. Native/naturalized.

15. Crinum jagus (Thomps.) Dandy, Journ. Bot. Lond. 77: 64 (1939). Amaryllis jagus Thomps., Bot. Displ. t. 6 (1798); C. giganteum Andr., Bot. Rep. t. 169 (1810).

Local name: Sukhdarshan. 
A bulbous, perennial herb. Flowers white, fragrant, perianth tube curved. Fruit a subglobose capsule. Fl. \& Fr.: April-July. Cultivated.

Specimens examined: Dhaka: Dhaka University Campus (Science Library), 3.5.2007, Sumona 25 (DUH); Dhaka University (Charukala Campus), 26.5.2007, Somona 39 (DUH).

16. Crinum latifolium L., Sp. Pl.: 291 (1753). C. zeylanicum L., Syst. ed. 12 (1767); C. moluccanum Roxb., Fl. Ind. 2: 140 (1832); C. ornatum Herb., Amaryll.: 262 (1837).

Local name: Sukhdarshan.

A bulbous, perennial herb. Flowers white, more or less streaked or tinged with red towards the centre, sometimes red-purple, nearly all over dorsal surface. Fruit a subglobose capsule, pinkish-maroon. Fl. \& Fr.: May-September. Native/naturalized.

Specimens examined: Dhaka: Dhaka University Botanical Garden, 28.4.2007, Somona 24 (DUH); Dhaka University Campus (Science Library), 19.9.2007, Sumona 44 (DUH).

17. Crinum pratense Herb., Amaryll.: 256 (1837). C. longifolium Roxb., Fl. Ind. 2: 130 (1832).

Local names: Bon peyaj, Sukhdarshan.

A bulbous, perennial herb. Flowers white, perianth lobes longer than the tube. Fruit a capsule. Fl. \& Fr.: May-August. Native/naturalized.

Specimen examined: Dhaka: Dhaka University Botanical Garden, 1.6.2000, Zashim Uddin 835 (DACB).

\section{Key to species}

Curculigo Gaertn., Fruct. 1: 63, t. 16 (1788).

1. Slender herbs; leaves small, linear; flowers in distichous spikes on scapes, hidden by the leaf-sheaths

\section{C. orchioides}

- $\quad$ Large stout herbs; leaves large, lanceolate, plicate; flowers on scapes projecting beyond the leaf-sheaths

C. latifolia

18. Curculigo latifolia [Dryand.] Ait., Hort. Kew. ed. 2, 2: 253 (1811). C. sumatrana Roxb., Fl. Ind. 2: 146 (1832); Molineria latifolia (Dryand.) Herb. ex Kurz, Tijds. Ned. Ind. 27: 232 (1864).

A large, stout herb. Flowers on scape projecting beyond the leaf-sheaths, unisexual (male) and bisexual, yellow. Fl. \& Fr.: May-October. Native/naturalized.

Specimen examined: Sylhet: Rema-Kalenga range, 24.5.1999, Zashim Uddin 719 (DACB). 
19. Curculigo orchioides Gaertn., Fruct. 1: 63, t. 16 (1788). C. brevifolia [Dryand.] Ait., Hort. Kew. ed. 2, 2: 253 (1811).

Local name: Talmuli.

A slender herb. Flowers distichous, hidden by the leaf-sheaths, the lowest bisexual, all the rest male, yellow. Fruit a capsule, 1-4-seeded. Seeds black. Fl. \& Fr.: JuneAugust. Native/naturalized.

Specimens examined: Bandarban: Kechua, 22.8.1987, Khan, Huq and Mia 7788 (DACB); Chittagong: Sitakund, 24.6.1979, Mia and Rahman 158 (DACB); 3.10.1940, S.K. Sen (DUH); Cox's Bazar: Goalmara Beat, 4.8.1990, Khan, Huq and Alam 8426 (DACB); Dinajpur: Singra (Sal forest), 25.8.1998, Mia et al. 4242 (DACB); Sylhet: Astagram, 21.4.1985, Huq and Mia 7032 (DACB).

Eucharis Planch. and Linden, Cat. no. 8: 3 (1853) et in Fl. des Serres,

Ser. 1, 8: 107 (1852-1853).

20. Eucharis grandiflora Planch. and Linden, Fl. des Serr. Ser. 1, 9: 255 (1853-1854).

A bulbous, perennial herb. Leaves lanceolate. Flowers white with staminal corona, waxy. Fl. \& Fr.: May-July. Cultivated.

Specimen examined: Dhaka: Dhaka University Campus (Science Library), 6.6.2007, Sumona 40 (DUH).

Gloriosa L., Syst. ed. 1 (1735); Sp. P1.: 305 (1753).

21. Gloriosa superba L., Sp. P1.: 305 (1753). G. simplex D. Don, Prod. Fl. Nep. 51 (1825).

Local names: Agnishikha, Bilambuli, Bishlanguli, Kalihari, Ulatchandal.

A climbing herb. Flowers axillary, greenish at first, then turning yellow, and in sequence to orange, scarlet and finally crimson. Fruit a capsule. Seeds sub-globose, orange. Fl. \& Fr.: July-November. Native/naturalized.

Specimens examined: Dhaka: Baldha Garden, 16.8.1966, Zeyauddin 223 (DUH); Govt. Nursery, 11.10.1970, M.A. Rahman 268 (DUH); Dhaka University Botanical Garden, 25.9.2007, Somona 47 (DUH). Dinajpur: Biral (Mayer pukur), 28.8.1998, Mia et al. 4392 (DACB). Gazipur: Zoydebpur, 28.6.1969, Subhash Chandra Ghosh 88 (DUH).

Haemanthus [Tourn.] L., Syst. ed. 1 (1735).

22. Haemanthus multiflorus Martyn ex Willd., Sp. P1. 2: 25 (1799). Scadoxus multiflorus Rafin., Fl. Tell. 5: 19 (1836).

Local names: Agni golock, Ball phul, May flower. 
A bulbous, perennial herb. Flowers reddish-pink. Fruit a berry, globose, red when ripe. Fl. \& Fr.: May-June (flowers usually in May). Cultivated.

Specimens examined: Dhaka: Dhaka University Botanical Garden, 28.5.1968, Mozahar 131 (DUH); Dhaka University Botanical Garden, 5.5.2007, Sumona 27 (DUH).

Hemerocallis L., Syst. ed. 1 (1735); Sp. Pl.: 324 (1753).

23. Hemerocallis fulva L., Sp. Pl. ed. 2: 462 (1764). H. disticha Donn, Hort. Contab. ed. 6: 93 (1811); H. longituba Miq., Ann. Mas. Bot. Lugd. Bat. 3: 152 (1867).

Local name: Komola lily.

A showy herb. Flowers orange. Fruit a loculicidal capsule. Seeds black, shining. Fl. \& Fr.: June-July. Cultivated.

Specimens examined: Dhaka: Dhaka University Botanical Garden, 28.5.1968, Mozahar 133 (DUH); Dhaka University Botanical Garden, 30.6.1970, Huq 81 (DACB); Dhaka University Botanical Garden, 12.5.2007, Sumona 33 (DUH).

Hippeastrum Herb., App. Bot. Reg.: 31 (1821).

24. Hippeastrum $\times$ johnsonii, a hybrid between $H$. reginae Herb. and H. vittatum Herb., Herbartia 15: 31-35 (1959).

Local name: Lal lily.

A herb with subterranean bulb. Flowers showy, funnel-shaped, more or less declinate, perianth red with white stripe towards the centre. Fl. \& Fr.: March-May. Cultivated.

Specimen examined: Dhaka: Dhaka University Campus (Science Library), 24.3.2007, Sumona 14 (DUH).

Hypoxis L., Syst. ed. 10: 986 (1759).

25. Hypoxis aurea Lour., Fl. Cochinch.: 200 (1790). H. minor D. Don, Prod. Fl. Nep. 53 (1825); Curculigo graminifolia Nimmo, J. Grah. Cat. Pl. Bomb. 215 (1839); Hypoxis franquevillii Miq., Fl. Ind. Bat. 3: 586 (1858).

A small, perennial herb. Inflorescence 1 or 2-flowered. Flowers yellow. Fruit a capsule. Seeds sub-globose, black, tuberculate. Fl. \& Fr.: May-August. Cultivated.

Molineria Colla, Hort. Repub. App. 2: 333, t. 18 (1826).

26. Molineria recurvata (Dryand.) Herb., Amaryll.: 84 (1834). Leucojum capitulatum Lour., Fl. Cochin. 199 (1790); Curculigo recurvata [Dryand.] Ait., Hort. Kew. ed. 2, 2: 253 (1811); C. capitulata (Lour.) O. Kuntze, Rev. Gen. 703 (1891). 
Local name: Satipata.

A stout herb. Flowers yellow. Ovary hardly produced upwards into a long beak or stipe. Fruit a berry, more than 20-seeded. Seeds black. Fl. \& Fr.: June-September. Native/naturalized.

Specimens examined: Dhaka: Dhaka University Botanical Garden, 5.9.2006, Sumona 2 (DUH). Cox's Bazar: Teknaf, 25.3.1973, M.M. Islam 792 (DUH); Teknaf, 16.6.1991, Khan et al. 8475 (DACB). Mymensingh: Haluaghat, 23.5.1989, Mia 2034 (DACB). Rangamati: Kaptai, 24.4.1997, Khan et al. 9835 (DACB). Sylhet: Lawachhera, 15.8.1976, Khan et al. 4227 (DACB).

Pancratium Dill. ex Linn., Syst. ed. 1 (1735).

\section{Key to species}

1. Staminal cup not bi-fid between the filaments

P. biflorum

- Staminal cup bi-fid between the filaments

2. Scape equal or longer than the leaves

- Scape shorter than the leaves
2

P. verecundum
P. triflorum

27. Pancratium biflorum Roxb., Fl. Ind. 2: 125 (1832). Pancratium longiflorum Buch.Ham. ex Roxb., Fl. Ind. 2: 125 (1832).

A perennial, bulbous herb. Flowers white with funnel-shaped, erose staminal corona. Fruit a capsule, 3-angled. Fl. \& Fr.: May-September. Cultivated.

Specimen examined: Dhaka: Baldha Garden, 23.3.2007, Sumona 12 (DUH).

28. Pancratium triflorum Roxb., Fl. Ind. 2: 126 (1832). P. malabathricum Herb., Amaryll.: 202, 206 (1837); Crinum pauciflorum Miq. ex Hook. f., Fl. Brit. Ind. 6: 285 (1892).

A perennial herb. Flowers white with broadly 2-toothed staminal corona between the filaments. Fl. \& Fr.: April-May. Native/naturalized.

Specimen examined: Khagrachari: Khagrachari, 6.4.2004, M. Yusuf 1602 (BCSIR Lab.).

29. Pancratium verecundum Ait., Hort. Kew. 1: 412 (1810).

Local names: Bakphul, Gor rashun.

A perennial, bulbous herb. Flowers spider-like, with 2-toothed staminal corona between the filaments. Fruit a sub-globose capsule. Seeds angled. Fl. \& Fr.: March-July. Native/naturalized.

Specimen examined: Dhaka: Dhaka University Botanical Garden, 5.2.2007, Sumona 7 (DUH). 
Proiphys Herb., App. [Bot. Reg.] 42 (1821).

30. Proiphys amboinensis (L.) Herb., App. [Bot. Reg. 7] 42 (1821). Pancratium amboinensis L., Sp. Pl.: 291 (1753); Eurycles sylvestris Salisb., Trans. Hort. Soc. 1: 337 (1812); E. amboinensis (L.) Lindl. in Loud., Enc. Pl. 242 (1829).

A herb with tunicated bulb. Leaves cordate-reniform. Flowers white, waxy. Staminal corona 6-partite. Fruit a globose berry. Fl. \& Fr.: April-August. Cultivated.

Specimens examined: Dhaka: Govt. Nursery, 26.4.1946, Sukhdeo (DUH); Dhaka University Botanical Garden, 25.5.1968, Mozahar 129 (DUH); Dhaka University Botanical Garden, 5.5.2007, Sumona 28 (DUH).

Urginea Steinh., Ann. Sc. Nat. Ser. 2, t. 14, 1: 322 (1834).

31. Urginea indica (Roxb.) Kunth, Enum. Pl. 4: 333 (1843). Scilla indica Roxb., Fl. Ind. 2: 147 (1832); S. coromandeliana Roxb., Fl. Ind. 2: 147 (1832); Urginea coromandeliana (Roxb.) Hook. f., Fl. Brit. Ind. 6: 347 (1892); U. senegalensis Kunth, Enum. P1. 4: 334 (1843).

Local names: Bon peyaj, Jongli peyaj, Kanda, Shamudra peyaj.

A perennial, bulbous herb. Flowers campanulate, greenish-white. Fruit a capsule, brownish-yellow. Seeds black, compressed. Fl. \& Fr.: February-May. Native/naturalized.

Specimen examined: Dhaka: Dhaka University Botanical Garden, 6.4.2007, Sumona 20 (DUH).

Zephyranthes Herb., App. [Bot. Reg.] 36 (1821).

\section{Key to species}

1. Leaves terete; spathe covering the ovary

- Leaves flat; spathe not covering the ovary

2. Spathe bi-fid

- $\quad$ Spathe not bi-fid

3. Perianth segments 6 or up to 8 , pink colour

- Perianth segments 6 , white but turn pink at maturity

\author{
Z. candida \\ 2 \\ 3 \\ Z. tubispatha \\ Z. grandiflora \\ Z. atamasco
}

32. Zephyranthes atamasco (Linn.) Herb., App. Bot. Reg.: 36 (1821). Amaryllis atamasco Blanco, Fl. Filip. 254 (1837).

Local name: Sada ghash-phul.

A perennial, bulbous herb. Flowers white but turn pink as it ages. Fruit a capsule. Fl. \& Fr.: April-May. Cultivated. 
Specimen examined: Dhaka: Dhaka University Botanical Garden, 11.4.2007, Sumona 21 (DUH).

33. Zephyranthes candida (Lindl.) Herb., Bot. Mag. 53: t. 2607 (1826). Amaryllis candida Lindl., Bot. Reg. (1825).

Local name: Sada ghash-phul.

A perennial, clump-forming, bulbous herb. Flowers white, spathe covering the ovary. Fruit a sub-globose capsule, yellowish-green. Seeds angular, flattened, testa black. Fl. \& Fr.: August-November. Cultivated.

Specimen examined: Dhaka: Dhaka University Campus (Science Library), 20.9.2007, Sumona 46 (DUH).

34. Zephyranthes grandiflora Lind1., Bot. Reg.: t. 902 (1825). Z. carinata Herb., Bot. Mag. t. 2594 (1825); Z. rosea Lindl., Bot. Reg. t. 821 (1825).

Local name: Golapi ghash-phul.

A bulbous, clump-forming, perennial herb. Flowers pink, spathe bi-fid. Fruit a capsule, deep green. Seeds angular, flattened, testa black. Fl. \& Fr.: June-October. Blooming soon after a heavy rainfall. Cultivated.

Specimen examined: Dhaka: Dhaka University Botanical Garden, 19.9.2007, Sumona 45 (DUH).

35. Zephyranthes tubispatha (L'Her.) Herb. ex Traub, Taxon 7: 110 (1958). Amaryllis tubispatha L'Her., Sert. Angl. 9 (1789); Z. nervosa Herb., Amaryll.: 172 (1837).

Local name: Holde ghash-phul.

A small, perennial herb. Flowers yellow. Fruit a capsule, yellowish-green. Seeds angular, flattened, testa black. Fl. \& Fr.: June-September. Native/naturalized.

Specimens examined: Dhaka: Dhaka University Botanical Garden, 10.4.1968, Mozahar 101 (DUH); Dhaka University Botanical Garden, 26.5.2007, Sumona 37 (DUH).

\section{Acknowledgements}

The authors are thankful to Dr. Alan Merrow, Research Geneticist and Systematist, National Germplasm Repository, Florida, the USA for identifying some critical materials. The authors are also grateful to Dr. Mahbuba Khanam, Director, Bangladesh National Herbarium for extending library facilities and Jahir Uddin Ahmed, Director, National Botanical Garden for his kind permission to observe and collect live specimens from Baldha Garden and National Botanical Garden. Our gratitude are also due to Dr. Mohammad Yusuf, Bangladesh Council of Scientific and Industrial Research Laboratory, Chittagong for supplying some specimens. 


\section{References}

Bentham, G. and Hooker, J.D. 1862-1883. Genera Plantarum, London.

Bessey, C.E. 1915. The phylogenetic taxonomy of flowering plants. Ann. Miss. Bot. Gard. 2: 109-164.

Engler, A. and Prantl, K. 1887-1915. Die Nat. Pflanz. ed. 2. Leipig.

Cowan, J.M. 1926. The flora of Chakaria Sundarbans. Rec. Bot. Surv. Ind. 11: 197-225.

Cronquist, A. 1981. An Integrated System of Classification of Flowering Plants. Columbia University Press, New York, pp. 1-1262.

Dassanayake, M.D. and Clayton, W.D. 1981. A Revised Handbook to the Flora of Ceylon. Oxford and IBH Co. Ltd., Calcutta, 14: 25-26.

Datta, R.M. and Mitra, J.N. 1953. Common plants in and around Dacca. Bul. Bot. Soc. Beng. 7(1\&2): 103105.

Deb, D.B. 1983. The Flora of Tripura State, Vol. 2. Today and Tomorrow's Printers and Publishers, New Delhi, India, pp. 1-602.

Heinig, R.L. 1925. List of Plants of Chittagong Collectorate and Hill Tracts. The Bengal Government Branch Press, Darjeeling, India, pp. 71-72.

Hooker, J.D. 1892. The Flora of British India. 23-A, Connaught Place, Dehra Dun, India 6: 227-362.

Hutchinson, J. 1934. The Families of Flowering Plants, Vol. 2. London, pp. 1-243.

Jackson, B.D. 1895 (rep. 1946). Index Kewensis (An Enumeration of the Genera and Species of Flowering Plants). Vols 1-2. Clardon Press, Oxford.

Lubna, A., Begum, R., Noor, S.S., Zaman, M.A. and Alam, S.S. 2004. Reversible flurescent chromosome banding in three Crinum spp. (Amaryllidaceae). Cytologia 69(1): 69-74.

Mia, M.M.K. and Khan, B. 1995. First list of Angiospermic Taxa of Bangladesh not included in Hooker's 'Flora of British India' and Prain's 'Bengal Plants'. Bangladesh J. Plant Taxon. 2(1\&2): 25-45.

Prain, D. 1903. Bengal Plants. Vol. 2. Indian reprint 1981. Bishen Singh Mahendra Pal Singh, Dehra Dun, India, pp. 663-1319.

Rahman, M.O. 2004. Second list of angiospermic taxa of Bangladesh not included in Hooker's 'Flora of British India' and Prain's 'Bengal Plants': Series-II. Bangladesh J. Plant Taxon. 11(2): 49-56.

Raizada, M.B. 1941. On the Flora of Chittagong. Indian Forester 67(5): 245-254.

Rendle, A.B. 1925 (rep. 1973). The classification of flowering plants, Vol. 1. Vikas Publishing House, India, pp. 1-413.

Sinclair, J. 1955. The flora of Cox's Bazar, East Pakistan. Bull. Bot. Soc. Bengal 9(2): 84-116.

Takhtajan, A. 1980. Outline of the classification of flowering plants (Magnoliophyta). Bot. Rev. 46: 225-359.

Uddin, M.Z. and Hassan, M.A. 2004. Flora of Rema-Kalenga Wildlife Sanctuary. IUCN, Bangladesh Country Office, Dhaka, Bangladesh, pp. 1-122.

Uddin, S.N., Khan, M.S., Hassan, M.A. and Alam, M.K. 1998. An annotated checklist of Sita Pahar at Kaptai in Bangladesh. Bangladesh J. Plant Taxon. 5(1): 13-46. 